\title{
Using Pseudoaffinity To Translation QFPP To LFPP
}

\author{
Basiya K. Abdulrahim \\ Department of Mathematics, College of Education \\ University of Garmian, Kurdistan Region -Iraq \\ Basiya.Kakawla@garmian.edu.krd
}

Tel: 07702492483

\begin{abstract}
In this paper, deal we with the problem of optimizing the ratio of two quadratic functions subject to a set linear constraints with the additional restriction that the optimal solution should also translation quadratic fractional programming problem (QFPP) to linear fractional programming problem (LFPP) by using pseudoaffinity after solving by modified simplex method. And consequently a convergent algorithm has been developed in the following discussion. Numerical examples have been provided to support the theory, by using Matlab 2016.
\end{abstract}

Keywords: Translation QFPP, by Pseudoaffinity to LFPP, Modified Simplex Method.

\subsection{Introduction}

The quadratic fractional programming problems (QFPP) are the topic of great importance in nonlinear programming. They are useful in many fields such as production planning, financial and corporative planning, health care and hospital planning. In various applications of nonlinear programming, one often encounters the problem in which the ratio of given two functions is to be maximized or minimized. Several methods to solve such problems are proposed in (1962) Charnes and Cooper ([6], [13]), Linear fraction problems (i.e. ratio of objectives that have numerator and denominator) have attracted considerable research and 
interest, their method depends on transforming this LFPP to an equivalent linear program, showed that by a simple transformation the original LFPP can be reduced to an LPP that can therefore be solved using a regular simplex method for LP. It was found that this approach is very useful for mathematicians because most theoretical results developed in LP could be relatively easily expanded to include LFPP [4]. Sing (1981) [11] did a useful study about the optimality condition in FP. In (2007) Tantawy studied a feasible direction method to solve LFPP [17]. Also in (2010) Salih studied and developed a feasible direction method to solve LFPP which is defined by Tantawy and we have suggested an approach to solve the same problem by using the modified simplex method [14]. Khurana and Arora (2011) studied an algorithm for solving a QFP when some of its constraints are homogeneous ([9], [10]). Moreover, in (2008) Fukushima and Hayashi have been addressed QFPP with quadratic constraints [8]. Abdulrahim, (2011) studies on solving QPP with extreme points [2]. In (2013) Abdulrahim, solving QFPP via feasible direction development and modified simplex method [3]. In (2013) Sulaiman and Nawkhass they have study a new modified simplex method to solve QFPP and compared it to a traditional simplex method by using pseudoaffinity of quadratic fractional functions [13]. Also in (2005) Biggs worked on Nonlinear Optimization with Financial Applications [5]. In (2013) Sulaiman and Nawkhass they have study a solving QFPP by using the Wolfe's method and a modified simplex approach [12]. To extend this work, we have been defined QFPP and investigated new technique to convert the quadratic fractional objective function to linear fractional objective function by using pseudoaffinity to generate the best compromising optimal solution. In addition, the special cases of the problem will be solved by Modified Simplex Method after convert the objective function to the pseudoaffinity function. 


\subsection{Definitions and Theorems}

\section{Definition 2.1: Linear Fractional Programming Problem}

The mathematical programming problem for LFPP can be formulated as follows:

Maximize (Minimize) $Z=\frac{\left(c^{T} x+y\right)}{\left(d^{T} x+\beta\right)}$

Subject to:

$$
x \in X=\left\{\begin{array}{r}
A X \leq b \\
x ; \quad A X \geq b \\
A X=b
\end{array}\right\}
$$

Where $x \in R^{n}, A$ is an $m \times n$ matrix; $c$ and $d$ are $n-$ vectors; $b \in R^{m}$ and $\gamma, \beta$ are scalar constants. Moreover $d^{T} x+\beta>0$ everywhere in $X$ [17].

\section{Definition 2.2: Quadratic Programming Problem}

If the optimization problem is of the form

Maximize (Minimize) $Z=\alpha+C^{T} x+\frac{1}{2} x^{T} G x$

Subject to :

$$
\begin{aligned}
& A x\left[\begin{array}{l}
\leq \\
\geq \\
=
\end{array}\right] b \\
& x \geq 0
\end{aligned}
$$

Where

$A=\left(a_{i j}\right)_{m \times n}$, matrix of cofficients, $\forall i=1,2, \ldots, m$, and $j=1,2, \ldots, n, b=\left(b_{1}, b_{2}, \ldots, b_{m}\right)^{T}$, $x=\left(x_{1}, x_{2}, \ldots, x_{n}\right)^{T}, C^{T}=\left(c_{1}, c_{2}, \ldots, c_{n}\right)^{T}$

, and $G=\left(g_{i i}\right)_{n \times n}$ is a positive definite or positive semi-definite symmetric square matrix, and $T$ is transposed then the constraints are linear and the objective function is quadratic. Such an optimization problem is said to be a QPP [1].

\section{Definition 2.3: Quadratic Fractional Programming Problem}

The mathematical programming problem for QFPP can be formulated as follows

Maximize (Minimize) $Z=\frac{\alpha_{1}+c_{1}^{T} x+\frac{1}{2} x^{T} G_{1} x}{\alpha_{2}+c_{2}^{T} x+\frac{1}{2} x^{T} G_{2} x}$

Subject to :

$$
\begin{aligned}
A x\left[\begin{array}{l}
\leq \\
\geq \\
=
\end{array}\right] b \\
x \geq 0
\end{aligned}
$$


Where $G_{1}, G_{2}$ are $(n \times n)$ matrix of coefficients with $G_{1}, G_{2}$ are symmetric matrixes. All vectors are assumed to be column vectors unless transposed(T), where $x$ is an $n$ dimensional vector of decision variables, $C_{1}, C_{2}$ is the $n$-dimensional vector of constants, $b$ is $m$-dimensional vector of constants, $\alpha_{1}, \alpha_{2}$ are scalars.

In this work the problem that has objective function is tried to be solved has the following form:

Maximize (Minimize) $Z=\frac{\left(c^{T} x+y\right)\left(e^{T} x+\delta\right)}{\left(d^{T} x+\beta\right)\left(f^{T} x+\varepsilon\right)}=\frac{\frac{\left(c^{T} x+p\right)\left(e^{T} x+\delta\right)}{\left(d^{T} x+\beta\right)}}{\left(f^{T} x+\varepsilon\right)}=\frac{\frac{\left(c^{T} x+y\right)\left(T_{x+\delta}\right)}{\left(f^{T}+\varepsilon\right)}}{\left(d^{T} x+\beta\right)}$ Subject to:

$$
\begin{aligned}
A x & {\left[\begin{array}{l}
\leq \\
\geq \\
=
\end{array}\right] b } \\
x & \geq 0
\end{aligned}
$$

Where $x \in R^{n}, A$ is an $m \times n$ matrix; $c_{y}, e_{,} d$ and $f$ are $n$-vectors; $b \in R^{m}$ and $\gamma, \beta, \delta, \varepsilon$ are scalar constants. Moreover $f^{T} x+\varepsilon, d^{T} x+\beta>0$ everywhere in $x$.

\section{Definition 2.4: Pseudoaffinity of Quadratic Fractional Functions}

In this section we are going to characterize the pseudoaffinity of quadratic fractional functions of the following kind:

$$
\begin{aligned}
& f(x)=\frac{\frac{\alpha_{1}+C_{1}^{T} x+\frac{1}{2} x^{T} G_{1} x}{\left(d^{T} x+\beta\right)}}{\left(f^{T} x+\varepsilon\right)}=\frac{g(x)}{\left(f^{T} x+\varepsilon\right)}=\frac{\frac{\left(c^{T} T_{x+\gamma\left(e^{T}\right.} T_{x+\varepsilon)}\right.}{\left(d^{T} x+\beta\right)}}{\left(f^{T} x+\varepsilon\right)} \\
& \text { Or } \left.f(x)=\frac{\frac{\alpha_{1}+C_{1}^{T} x+\frac{1}{2} x^{T} G_{1} x}{f^{T}}}{\left(d^{T} x+\beta\right)}=\frac{g(x)}{\left(d^{T} x+\beta\right)}=\frac{\frac{\left(c^{T} x+\eta\left(\varepsilon^{T} T_{x+\delta}\right.\right.}{\left(f^{T}\right)}}{\left(d^{T} x+\beta\right)}\right)
\end{aligned}
$$

Defined on the set $X=\left\{x \in \Re^{n}:\left(d^{T} x+\beta\right)>0\right\}$, or $X=\left\{x \in \Re^{n}:\left(f^{T} x+\varepsilon\right)>0\right\}$ where $G_{1} \neq 0$ is a $n \times n$ symmetric matrix, $d, f, x, C_{1} \in \Re^{n}, f, d \neq 0$, and $\alpha_{1}, \beta, \varepsilon, \delta, \gamma \in \Re$. Note that being $G_{1}$ symmetric, it is $G_{1} \neq 0$ if and only if $v_{0}\left(G_{1}\right) \leq n-1[7]$.

Corollary 2.1: Consider function $f(x)$ defined in (1) and suppose that there exist $a_{0}, b_{0}, p_{0} \in \Re, a_{0} \neq 0$, such that $f(x)$ can be written in the following form: 


$$
\begin{gathered}
f(x)=\frac{a_{0} d^{T} x+b_{0}+\frac{a_{0} p_{0}}{\left(d^{T} x+\beta\right)}}{\left(f^{T} x+\varepsilon\right)} \\
\text { or } f(x)=\frac{a_{0} f^{T} x+b_{0}+\frac{a_{0} p_{0}}{\left(f^{T} x+\varepsilon\right)}}{\left(d^{T} x+\beta\right)}
\end{gathered}
$$

i) If $p_{0} \leq 0$ then $g(x)$ is pseudoaffine on $X$.

ii) If $p_{0}>0$ then $g(x)$ is pseudoaffine on

$$
X_{1}=\left\{x \in \Re^{n}:\left(d^{T} x+\beta\right)>\sqrt{p_{0}}\right\} \text { and } X_{2}=\left\{x \in \Re^{n}: 0<\left(d^{T} x+\beta\right)<\sqrt{p_{0}}\right\}[7] \text {. }
$$

In our studies we take special cases where $p_{0}=0$, then the function $g(x)=a_{0} d^{T} x+b_{0}$ is pseudoaffine on $X, f(x)=\frac{a_{0} d^{T} x+b_{0}}{\left(f^{T} x+\varepsilon\right)}$ but where $p_{0}>0$, then the function $g(x)=a_{0} d^{T} x+b_{0}+\frac{a_{0} p_{0}}{\left(d^{T} x+\beta\right)}$ is not pseudoaffine on $X, f(x)=\frac{a_{0} d^{T} x+b_{0}+\frac{a_{0} p_{0}}{\left(a^{T} x+\beta\right)}}{\left(f^{T} x+s\right)}$ and linear fractional functions by adding constraints respectively then it can solve it by Modified Simplex Method, which is shown in numerical examples and result section 1.5.

\subsection{Modified Simplex Method Development}

Simplex method is developed by Dantzig in (1947). The Simplex method provides a systematic algorithm which consists of moving from one basic feasible solution (one vertex) to another in prescribed manner such that the value of the objective function is improved. This procedure of jumping from vertex to vertex is repeated. If the objective function is improved at each jump, then no basis can ever be repeated and there is no need to go to back to vertex already covered. Since the number of vertices is finite, the process must lead to the optimal vertex in a finite number of steps. The Simplex algorithm is an iterative (step by step) procedure for solving linear programming problems. It consists of:

I. Having a trail basic feasible solution to constraint equations.

II. Testing whether is an optimal solution.

III. Improving the first trial solution by a set of rules, and repeating the process till an optimal solution is obtained. For more details [15]. 
Modified Simplex method to solve linear fractional programming problem and to solve quadratic objective function can be written as the produced two linear functions (QPP) [16]. Using modified Simplex method to solve the numerical example to apply simplex process [16]. First we find $\Delta_{j 1}$, and $\Delta_{j 2}$ from the coefficient of numerator and denominator of objective function respectively, by using the following formula:

$\Delta_{j i}=C_{j i}-C_{B i} x_{j i}, i=1,2, j=1,2, \ldots, m+n$, $Z_{1}=C_{B 1} V_{B}+\gamma, Z_{2}=C_{B 2} V_{B}+\beta, \gamma, \beta$ are constants, $Z=\frac{z_{1}}{z_{2}}$

In this approach we define the formula to find $\Delta_{j}$ from $Z_{1}, Z_{2}, \Delta_{j 1}$ and $\Delta_{j 2}$ as follows: $\Delta_{j}=Z_{2} \Delta_{j 1}-Z_{1} \Delta_{j 2}$. Here $C_{j i}$ are the coefficients of the basic and non-basic variables in the objective function and, $C_{B i}$ are the coefficients of the basic variables in the objective function, $j=1,2, \ldots, m+n, i=1,2$. For testing optimality solution must be all $\Delta_{j} \leq 0$ but here all $\Delta_{j}$ not lesser than zero, and then the solution is not optimal. Repeat the same approach to find next feasible solution.

\subsection{Algorithm For Modified Simplex Method Of QFPP}

An algorithm for solving QFPP by modified simplex method can be summarized as follows ([14], [16]):

Step1: Write the standard from of the problem, by introducing slack, Surplus and artificial variables to constraints, and write starting simplex table, after convert the quadratic fractional objective function to linear fractional objective function by using pseudoaffinity.

Step2: Write the $\Delta_{j}$ row in the starting simplex table as: $\Delta_{j}=Z_{2} \Delta_{j 1}-Z_{1} \Delta_{j 2}$

Step3: Use simplex process to find the solution.

Step4: Check the feasibility of the solution in step3, if is feasible then go to step5, otherwise use dual simplex method to remove infeasibility.

Step5: Check the solution for optimality if all $\Delta_{j} \leq 0$, then the solution is optimal, otherwise go to step3. 


\subsection{Numerical Example and Results}

Example 1: We consider the following QFPP as

$\operatorname{Max} \cdot Z=\frac{9 x_{1}^{2}+24 x_{1} x_{2}+16 x_{2}^{2}+6 x_{1}+8 x_{2}+1}{3 x_{1}^{2}+7 x_{1} x_{2}+4 x_{2}^{2}+7 x_{1}+9 x_{2}+2}$

Subject to:

$$
\begin{aligned}
& x_{1}+x_{2} \leq 6 \\
& 8 x_{1}+4 x_{2} \leq 32 \\
& x_{1} \leq 3 \\
& x_{2} \leq 5 \\
& x_{1}, x_{2} \geq 0
\end{aligned}
$$

Solution 1: Solving the example 1 by Modified Simplex Method, after convert the objective function to pseudoaffinity function as follows:

$\operatorname{Max} . Z=\frac{\frac{9 x_{1}^{2}+24 x_{1} x_{2}+16 x_{2}^{2}+6 x_{1}+8 x_{2}+1}{\left(\mathrm{sx}_{1}+4 x_{2}+1\right)}}{\left(x_{1}+x_{2}+2\right)}$ Where $G_{1}=\left[\begin{array}{ll}18 & 24 \\ 24 & 32\end{array}\right], C_{1}^{T}=\left[\begin{array}{l}6 \\ 8\end{array}\right], d^{T}=\left[\begin{array}{l}3 \\ 4\end{array}\right], \alpha_{1}=1, \beta=1$

Then we get $g(x)=3 x_{1}+4 x_{2}+1$ is pseudoaffinity function by corollary part (i) where $p_{0}=0$

And we get $\operatorname{Max} . Z=\frac{3 x_{1}+4 x_{2}+1}{x_{1}+x_{2}+2}$. After finding the values of the objective function by

\begin{tabular}{|c|c|c|c|c|c|c|c|c|c|c|}
\hline & \multirow{2}{*}{$\begin{array}{l}C_{j 1} \\
C_{j 2}\end{array}$} & \multirow{2}{*}{$\begin{array}{l}3 \\
1 \\
\end{array}$} & \multirow{2}{*}{$\begin{array}{l}4 \\
1\end{array}$} & \multirow{2}{*}{$\begin{array}{l}0 \\
0\end{array}$} & \multirow{2}{*}{$\begin{array}{l}0 \\
0\end{array}$} & \multicolumn{3}{|c|}{0} \\
\hline & & & & & & & & \multicolumn{2}{|c|}{$\begin{array}{ll}0 & 0 \\
\end{array}$} & \\
\hline B.V & $C_{B 1}$ & $C_{B 2}$ & $V_{B}$ & $x_{1}$ & $x_{2}$ & $x_{3}$ & $x_{4}$ & $x_{5}$ & $x_{6}$ & Min ratio \\
\hline$x_{3}$ & 0 & 0 & 6 & 1 & 1 & 1 & 0 & 0 & 0 & $6 / 1=6$ \\
\hline$x_{4}$ & 0 & 0 & 32 & 8 & 4 & 0 & 1 & 0 & 0 & $32 / 4=8$ \\
\hline$x_{5}$ & 0 & 0 & 3 & 1 & 0 & 0 & 0 & 1 & 0 & \\
\hline$x_{6}$ & 0 & 0 & 5 & 0 & 1 & 0 & 0 & 0 & 1 & $5 / 1=5$ \\
\hline$Z_{1}=$ & & & $\Delta_{j 1}$ & 3 & 4 & 0 & 0 & 0 & 0 & \\
\hline$z_{2}=$ & & & $\Delta_{j 2}$ & 1 & 1 & 0 & 0 & 0 & 0 & \\
\hline & $\frac{1}{2}=\frac{1}{2}$ & & $\Delta_{j}$ & 5 & 7 & 0 & 0 & 0 & 0 & \\
\hline
\end{tabular}
Modified Simplex Method with used the same constraints, After 2 steps we obtained the initial table as follows in table 1. After three iterations, we obtained the result in the following table 2:

\section{Table 1: Initial table for example 1 by Modified Simplex Method}


Table 2: Final table for example 1 by Modified Simplex Method

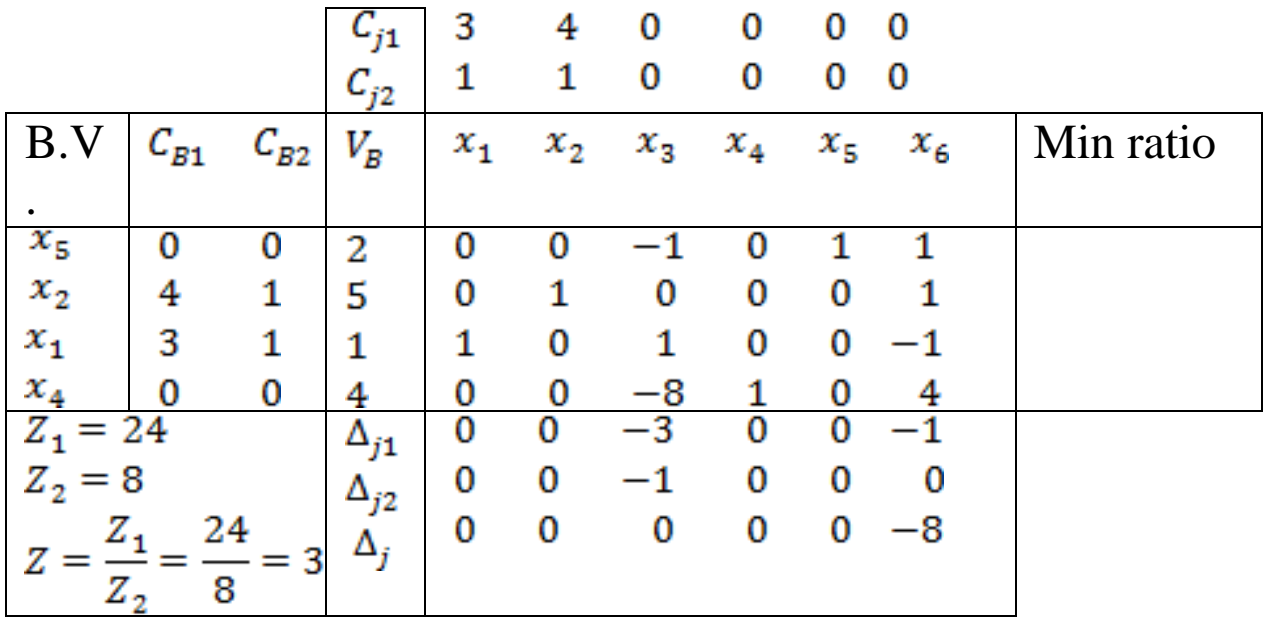

After solving it by Modified Simplex Method, we get $\operatorname{Max} . Z=3$ and $x_{1}=1, x_{2}=5$

Example 2: We consider the following QFPP as

$\operatorname{Max} . Z=\frac{-8 x_{1}^{2}+24 x_{2}^{2}+32 x_{a}^{2}+16 x_{1} x_{2}+64 x_{2} x_{g}+16 x_{2}+16 x_{g}+2}{16 x_{1}^{2}+16 x_{2}^{2}+32 x_{n}^{2}+32 x_{1} x_{2}+48 x_{2} x_{g}+48 x_{1} x_{g}+24 x_{1}+24 x_{2}+40 x_{g}+8}$

Subject to:

$$
\begin{aligned}
& x_{1}+3 x_{2}+2 x_{3} \leq 9 \\
& 3 x_{1}+2 x_{2}+x_{3} \leq 8 \\
& 2 x_{1}+x_{2}+3 x_{3} \leq 7 \\
& x_{1}, x_{2}, x_{3} \geq 0
\end{aligned}
$$

Solution 2: Solving the example (2) by Modified Simplex Method, after convert the objective function to pseudoaffinity function as follows:

$\operatorname{Max} . Z=\frac{\frac{-\mathrm{gx}_{1}^{\mathrm{N}}+24 x_{2}^{2}+\operatorname{sn} x_{g}^{2}+16 x_{1} x_{2}+64 x_{2} x_{g}+16 x_{2}+16 x_{g}+2}{\left(\mathrm{gx}_{1}+8 x_{2}+16 x_{g}+4\right)}}{\left(2 x_{1}+2 x_{2}+2 x_{g}+2\right)}$

Where $G_{1}=\left[\begin{array}{ccc}-16 & 16 & 0 \\ 16 & 48 & 64 \\ 0 & 64 & 64\end{array}\right], C_{1}^{T}=\left[\begin{array}{c}0 \\ 16 \\ 16\end{array}\right], d^{T}=\left[\begin{array}{c}8 \\ 8 \\ 16\end{array}\right], \alpha_{1}=2, \beta=4$

Then we get $g(x)=-x_{1}+3 x_{2}+2 x_{3}+\frac{1}{2}$ is pseudoaffinity function by corollary part (i) where $p_{0}=0$. And we get $\operatorname{Max} . Z=\frac{-x_{1}+3 x_{2}+2 x_{x}+\frac{1}{z}}{2 x_{1}+2 x_{2}+2 x_{x}+2}$. After finding the values of the objective function by Modified Simplex Method with used the same constraints, After 2 steps we obtained the initial table as follows in table 1. After two iterations, we obtained the result in the following table 2: 
Table 1: Initial table for example 2 by Modified Simplex Method

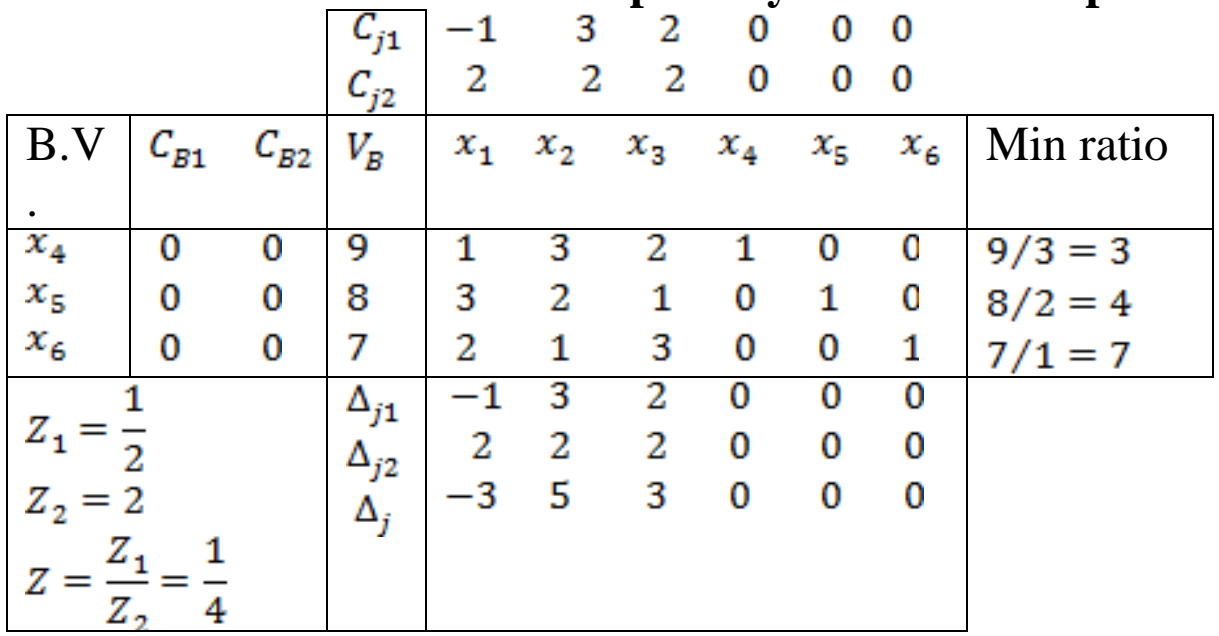

Table 2: Final table for example 2 by Modified Simplex Method

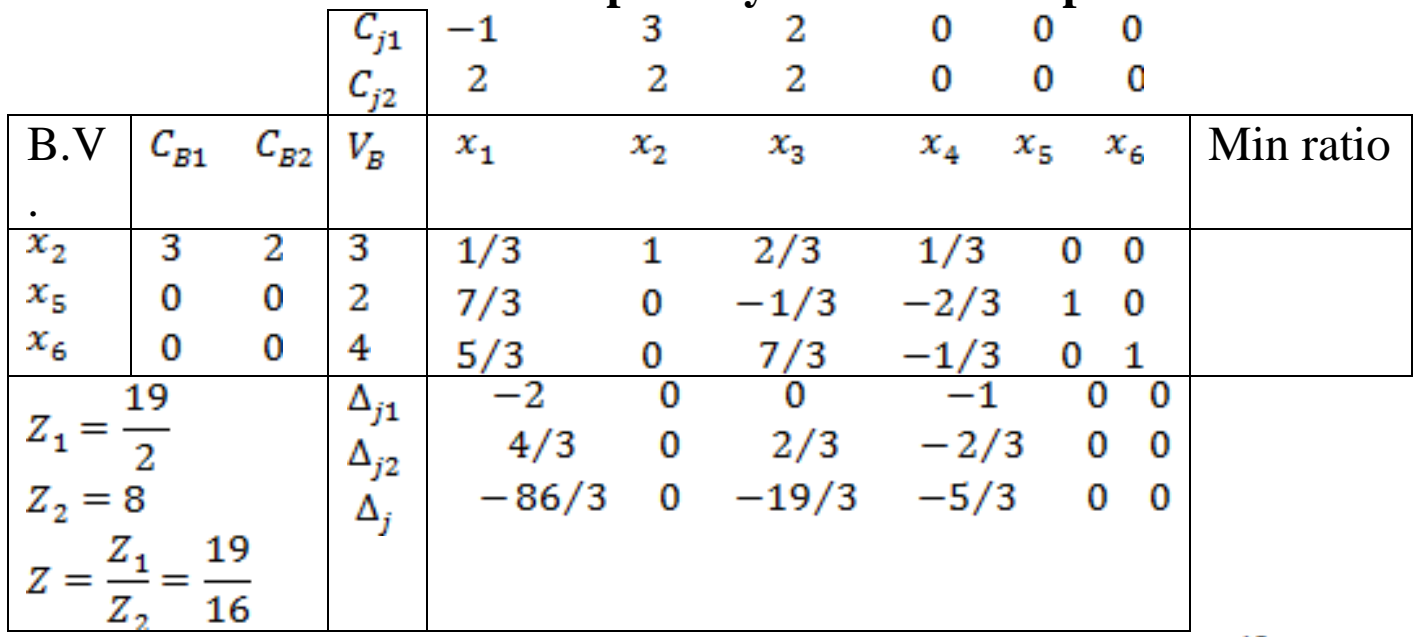

After solving it by Modified Simplex Method, we get $\operatorname{Max} . Z=\frac{19}{16}$ and $x_{1}=0$,

$x_{2}=3, x_{3}=0$

Example 3: We consider the following QFPP as

Max. $Z=\frac{4 x_{1}^{n}+4 x_{2}^{2}+8 x_{1} x_{2}+9 x_{1}+9 x_{2}+3}{2 x_{1}^{n}+2 x_{2}^{2}+4 x_{1} x_{2}+5 x_{1}+5 x_{2}+2}$

Subject to:

$$
\begin{aligned}
& 4 x_{1}-2 x_{2} \leq 20 \\
& 3 x_{1}+5 x_{2} \leq 25 \\
& x_{1}, x_{2} \geq 0
\end{aligned}
$$

Solution 3: Solving the example 3 by Modified Simplex Method, after convert the objective function to pseudoaffinity function as follows: 
$\operatorname{Max} . Z=\frac{\frac{4 x_{1}^{2}+4 x_{2}^{2}+8 x_{1} x_{2}+9 x_{1}+9 x_{2}+g}{\left(x_{1}+x_{2}+2\right)}}{\left(2 x_{1}+2 x_{2}+1\right)}$ Where $G_{1}=\left[\begin{array}{ll}8 & 8 \\ 8 & 8\end{array}\right], C_{1}^{T}=\left[\begin{array}{l}9 \\ 9\end{array}\right], d^{T}=\left[\begin{array}{l}1 \\ 1\end{array}\right], \alpha_{1}=3, \beta=2$

Then we get $g(x)=\left(4 x_{1}+4 x_{2}+1\right)+\frac{1}{\left(x_{1}+x_{2}+2\right)}$ is pseudoaffinity function by corollary part (ii) where $p_{0}>0$. And we get

$$
\operatorname{Max} . Z=\frac{\left(4 x_{1}+4 x_{2}+1\right)+\frac{1}{\left(\left(x_{1}+x_{2}+2\right)\right.}}{\left(2 x_{1}+2 x_{2}+1\right)}=\frac{\left(4 x_{1}+4 x_{2}+1\right)}{\left(2 x_{1}+2 x_{2}+1\right)}+\frac{\frac{1}{\left(2 x_{1}+x_{2}+2\right)}}{\left(2 x_{1}+2 x_{2}+1\right)}
$$

Here we have the remainder second part $c=(0,0) d=(1,1), \alpha=1, \beta=2$

$\left(c-\frac{\alpha}{\beta} d\right) x+\frac{\alpha}{\beta}=\left[(0,0)-\left(\frac{1}{2}\right)(1,1)\right]\left(\begin{array}{l}x_{1} \\ x_{2}\end{array}\right)+\left(\frac{1}{2}\right)=-\frac{1}{2} x_{1}-\frac{1}{2} x_{2}+\frac{1}{2}$

$\operatorname{Max} . Z=\frac{\left(4 x_{1}+4 x_{2}+1\right)}{\left(2 x_{1}+2 x_{2}+1\right)}+\frac{\left(-\frac{1}{2} x_{1}-\frac{1}{2} x_{2}+\frac{1}{2}\right)}{\left(2 x_{1}+2 x_{2}+1\right)}=\frac{\frac{7}{2} x_{1}+\frac{7}{2} x_{2}+\frac{\pi}{2}}{\left(2 x_{1}+2 x_{2}+1\right)}$

After finding the values of the objective function by Modified Simplex Method with used the same constraints After 2 steps we obtained the initial table as follows in table 1. After two iterations, we obtained the result in the following table 2:

Table 1: Initial table for example 3 by Modified Simplex Method

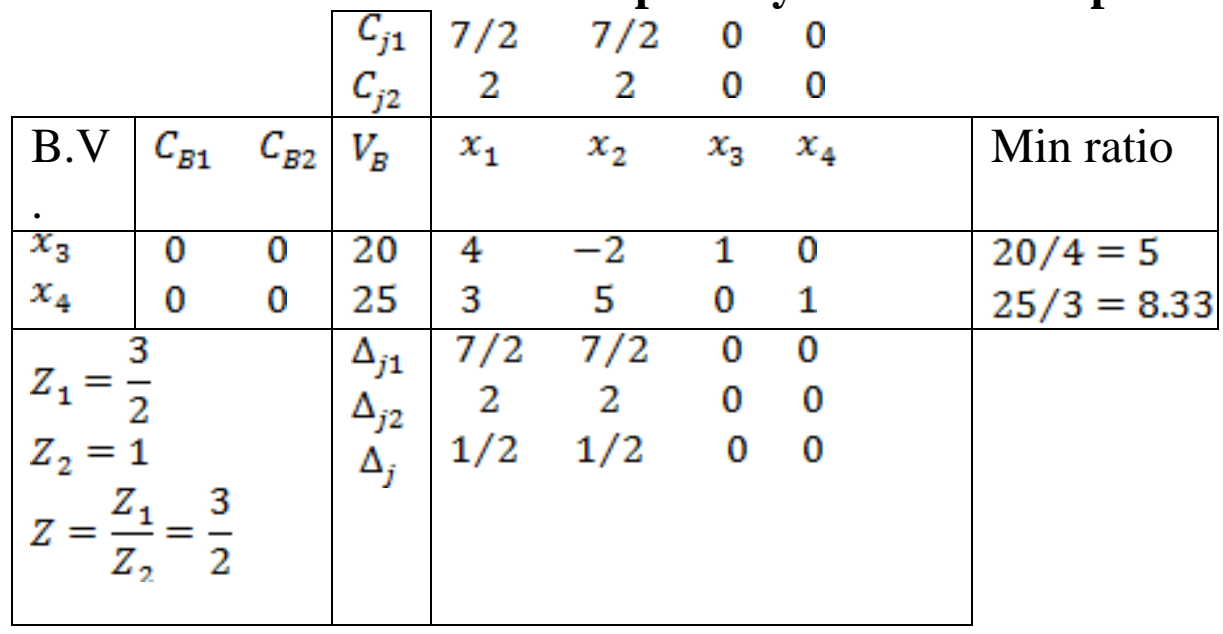


Table 2: Final table for example 3 by Modified Simplex Method

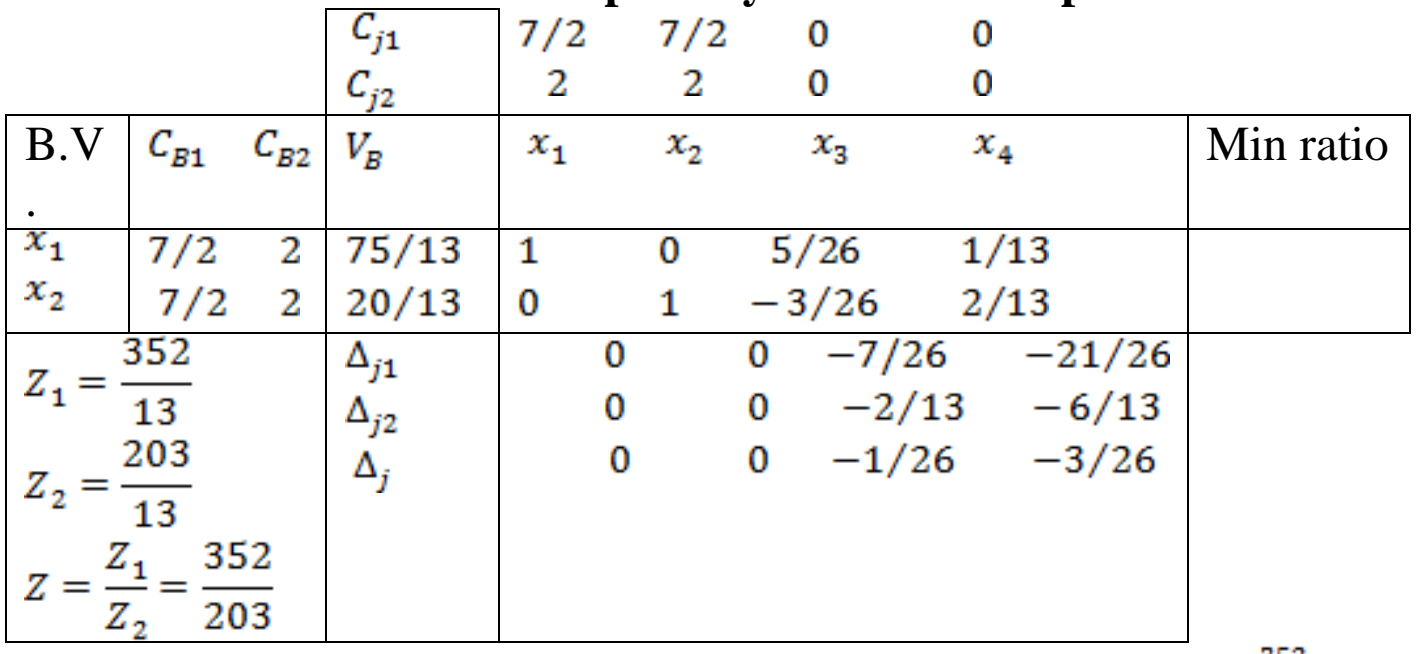

After solving it by Modified Simplex Method, we get $\operatorname{Max} . Z=\frac{352}{203}$ and $x_{1}=\frac{75}{13^{\prime}}$ $x_{2}=\frac{20}{13}$

\subsection{Comparison of the Numerical Results}

Now, we are going to comparison the numerical results which are obtained of the examples as below in table 3 :

Table 3: Comparison between results of the Objective Functions

\begin{tabular}{|l|l|l|}
\hline Examples & $\begin{array}{c}\text { Before Correct The Objective } \\
\text { Function (QFPP) }\end{array}$ & $\begin{array}{c}\text { After Correct The Objective } \\
\text { Function (LFPP) }\end{array}$ \\
\hline Example1 & Max. $Z=3$ and $x_{1}=1, x_{2}=5$ & Max. $Z=3$ and $x_{1}=1, x_{2}=5$ \\
\hline Example2 & Max. $Z=\frac{19}{16}$ and $x_{1}=0, x_{2}=3, x_{3}=0$ & Max. $Z=\frac{19}{16}$ and $x_{1}=0, x_{2}=3, x_{3}=0$ \\
\hline Example3 & Max. $Z=\frac{3705}{1907}$ and $x_{1}=\frac{75}{13}, x_{2}=\frac{20}{13}$ & Max. $Z=\frac{352}{203}$ and $x_{1}=\frac{75}{13}, x_{2}=\frac{20}{13}$ \\
\hline
\end{tabular}

In the above table 3 , we compare the result. It is notice that value of objective function in example 1, example 2, and example 3 they have same results when it 
solved by Modified Simplex Method after convert the objective function by Pseudoaffinity function.

\subsection{Discussion}

In this paper solved QFPP by the Modified Simplex Method after convert objective function by Pseudoaffinity function to found the maximum value of QFPP. The optimal solution must be at one of the points of the polygon of the feasible, sometimes it may be need to use corollary 2.2 part (ii) for finding best optimal solution for the problem. The comparison of this method is based on the value of the objective function, the study found that Max.Z resulted of that method are same, therefore we can solve of QFPP by this method under our method and algorithm. Consequently reliable results have been found.

\subsection{Acknowledgments}

My thanks are to all of the staff of the College of Education, University of Garmian in Kalar. My heartfelt thanks go to all those who helped me especially staff of the Conference, University of Garmian in Kalar-Garmian. Finally, I would like to thank all the members of my family for their encouragement, help and support.

\section{References}

1. Antoniou, A. Wu-Sheng Lu., (2007) "Practical Optimization Algorithms and Engineering Applications", Department of Electrical and Computer Engineering University of Victoria, Canada, Springer Science+ Business Media, LLC, P (675).

2. Abdulrahim, B. K., (2011) "On Solving Quadratic Programming Problem with Extreme Points", M.Sc. Thesis, University of Sulaimani, Sulaimani/Iraq.

3. Abdulrahim, B. K., (2013) "Solving Quadratic Fractional Programming Problem via Feasible Direction Development and Modified Simplex Method", Journal of Zankoy Sulaimani - Part A (JZS-A), for Pure and Applied Science, A Scientific Journal Issued by the University of Sulaimani, Kurdistan Region-Iraq, Vol. 15A, No. 2, PP.45-52, ISSN 1812-4100. 
4. Bajalinov, E. B. (2003) "Linear Fractional Programming, Theory Methods Applications and Software", Kluwer Academic Publishers.

5. Biggs, M. B., (2005) "Nonlinear Optimization with Financial Applications. Kluwer Academic Publishers, England, P (41).

6. Charnes, A., and Cooper, W.W., (1962) "Programming with Linear Fractional Functions", Nava research Quarterly, Vol.9, No.3-4, and PP.181-186.

7. Cambini, R., and Carosi, L., ( 2001) "On the Pseudoaffinity of a class of Quadratic Fractional Functions", Department of Statistics and Applied Mathematics, University of Pisa, Pisa, Giugno, Stampato in Proprio, P (17).

8. Fukushima, M., and Hayashi, Sh., (2008) "Quadratic Fractional Programming Problems with Quadratic Constraints", Department of Applied Mathematics and Physics, Graduate School of Informatics, Kyoto University.

9. Khurana, A., and Arora, S. R., (2011) "A Quadratic Fractional Programming with Linear Homogenous Constraints", African Journal of Mathematics and Computer Science, Research, Vol. 4, No. 2, PP. 84-92.

10. Khurana, A., and Arora, S. R., (2011) "An Algorithm for Solving Quadratic Fractional Program with Linear Homogeneous Constraints", Vietnam Journal of Mathematics, Vol. 39, No. 4, PP. 391-404.

11. Sing, H. C., (1981) "Optimality Condition in Functional Programming", Journal of Optimization Theory and Applications, Vol. 33, PP.287-294.

12. Sulaiman, N. A., and Nawkhass, M. A., (2013) "Solving Quadratic Fractional Programming Problem", International Journal of Applied Mathematical Research, Vol. 2 No. 2, PP. 303-309, DOI 10.14419/ijamr.v2i2.838.

13. Sulaiman, N. A., and Nawkhass, M. A., (2013) "A New Modified Simplex Method to Solve Quadratic Fractional Programming Problem and Compared it to a Traditional Simplex Method by Using Pseudoaffinity of Quadratic Fractional Functions", HIKARI Ltd, Applied Mathematical Sciences, Vol. 7, No. 76, PP. 3749 - 3764 ,http://dx.doi.org/10.12988/ams.2013.36298. 
14. Salih, A. D., (2010) "On Solving Linear Fractional Programming Problems with Extreme Points", M.Sc. Thesis, University of Salahaddin, Hawler/Iraq 15. Sharma, S. D., (1988) "Operations Research”, Kedar Nath Ram Nath BCO., Meerut, India, P (559).

16. Sharma, S. D., (1980) "Nonlinear and Dynamic Programming”, Kedar Nath Ram Nath and CO., Meerut, India, P (547).

17. Tantawy, S. F., (2007) "Using Feasible Directions to solve Linear Programming Problems", Australian Journal of Basic and Applied Science, Vol. 1, No. 2, PP. 109-114, ISSN 1991-8178.

\section{الخلاصة}

في هذا البحث، نتعامل مع نسبة أمثلية لأثنيز من الدوال من اللرجة الثانية للقيود المجموعة الخطية و ايضا تحويل مشكلة كسور البرمجة من الدرجة الثانية الى مشكلة كسور البرمجة الخطية بأستخدام Pseudoaffinity وحلها بطريقة المبسطة المتطورة. و بالتالي تم تطوير خوارزمية متقاربة في المناقشة التالية. و قد تم تزويلد أمثلة عددية لهذه النظرية بأستخدام ماتلاب 2016.

\section{يوخته}

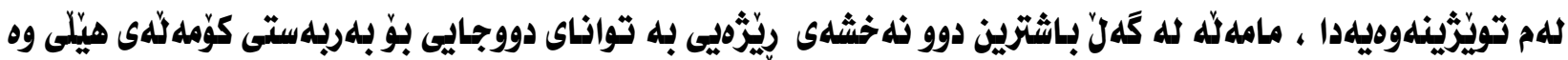

هله ودها كَوْرينى كيّشهى كلهتى توانى دووجايى بوّ كيّشهى كهرتى هيّلى به كارهيّنانى Pseudoaffinity وه

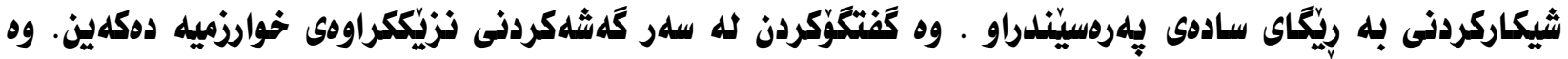

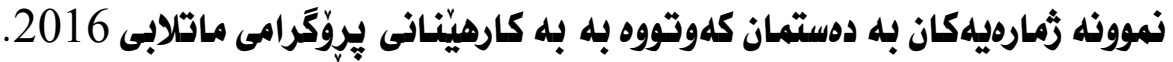

